\title{
One Fusion Approach of Fault Diagnosis Based on Rough Set Theory and Dezert-Smarandache Theory
}

\author{
Yanqin Su ${ }^{1, a}$, Zhenyu Song ${ }^{2}$, Tingxue $\mathrm{Xu}^{3}$ \\ ${ }^{1}$ Gratduate Student's, Naval Aeronautical and Astronautical University, Yantai Shandong, China \\ ${ }^{2}$ Department of Science Research, Naval Aeronautical and Astronautical University, Yantai \\ Shandong, China \\ ${ }^{3}$ Department of Ordnance Science and Technology, Naval Aeronautical and Astronautical University, \\ Yantai Shandong, China \\ asuyq1982@163.com
}

Key words: Rough Sets Theory; Dezert-Smarandache Theory; fault diagnosis

\begin{abstract}
There are the advantage of Rough Sets Theory and evidence theory for processing uncertain information, and one fusion approach of fault diagnosis based on the Rough Sets Theory and Dezert-Smarandache Theory. Firstly the abundant condition attribution was reduced through Rough Sets Theory, then evidence combining results of each reduced result were calculated through the basic probability assignment and normalized attribution significance. The diagnosis results were combined by DSmT combining equation. Finally the above method was applied to some equipment diagnosis to verify its effectiveness.
\end{abstract}

\section{Introduction}

The equipment fault diagnosis is important for equipment comprehensive support so the suited fault diagnosis methods research aiming at the equipment characteristic is very necessary. Generally, the equipment diagnosis information is uncertain, incorrect and incomplete, so the fault diagnosis is a uncertain reasoning and decision processing actually ${ }^{[1]}$.

Rough Sets Theory has the advantage of processing uncertain, incorrect and incomplete data, and evidence theory is an effective uncertain reasoning method, so fusion research based on these two methods is more effective and correct for fault diagnosis. Relative to Dempster-Shafer Theory, Dezert-Smarandache Theory (DSmT) is a new evidence theory in 1992, which can express and process the uncertain and conflict information better and combine uncertain, high conflict and incorrect evidence resource expressed by belief function.

\section{Rough Sets Theory}

In 1982, Rough Sets Theory is proposed by Polish mathematician Z. Pawlak ${ }^{[2]}$. Rough Sets Theory is defined in the upper approximation set and lower approximation. Suppose $U$ is the domain and $R$ is the equivalence relation of $U, P=(U, R)$ is named as Pawlak approximation space. $\forall X \subseteq U$,

$$
\begin{gathered}
\underline{R}(X)=\left\{x \in U \mid[x]_{R} \subseteq X\right\}=\bigcup\left\{[x]_{R} \mid[x]_{R} \subseteq X\right\} \\
\bar{R}(X)=\left\{x \in U \mid[x]_{R} \bigcap X \neq \Phi\right\}=\bigcup\left\{[x]_{R} \mid[x]_{R} \bigcap X \neq \Phi\right\}
\end{gathered}
$$

as the upper approximation set and lower approximation of $X$ to the approximation space $(U, R)$, in which, $B N_{R}(X)=\bar{R}(X)-\underline{R}(X)$ is $R$ boundary region of $X, P O S_{R}(X)=\underline{R}(X)$ is $R$ positive region of $X, N E G_{R}(X)=U-\bar{R}(X)$ is $R$ negative region of $X$.

In Rough Sets Theory, the quad system $S=(U, A, V, f)$ is a knowledge representation system, in which $U$ is the domain, $A$ is attribute set, $V=\bigcup_{\alpha \in A} V_{\alpha}$, and $V_{\alpha}$ is the range of the property $\alpha$. $f: U \times A \rightarrow V$ is an information function, it gives each attribute of each object an information value ${ }^{[3]} . M_{n \times n}$ is defined as relative core combined with all simple attribution, 


$$
C O R E_{C}(D)=\left\{\alpha \mid(\alpha \in C) \wedge\left(\exists c_{i j},\left(\left(c_{i j} \in M_{n \times n}\right) \wedge\left(c_{i j}=\{\alpha\}\right)\right)\right)\right\}
$$

The dependence degree between condition attribution $C$ and decision attribution $D$ is defined as

$$
v(C, D)=\frac{\operatorname{card}\left[\operatorname{pos}_{C}(D)\right]}{\operatorname{card}(U)}
$$

In which, $\operatorname{pos}_{C}(D)=\bigcup_{X \in U / D} \frac{C}{D}(X)$ is positive region of $D$ in $\operatorname{IND}(C), \operatorname{card}(\bullet)$ is the set cardinality. The attribution importance degree is defined as $S G F(a, R, D)=\gamma(R \bigcup\{a\}, D)-\gamma(R, D)$.

\section{DSmT}

In 1967, Dempster proposed the evidence theory, then Shafer expanded and developed it, so the evidence theory is also called D-S Theory.In 2002, Dezert and Smarandache proposed DSmT ${ }^{[4-5]}$.

Define a basic When the basic probability assignment function $m: D^{U} \rightarrow[0,1]$ is relative with the given evidence resource, that is

$$
m(\phi)=0, \sum_{A \in D^{U}} m(A)=1
$$

Suppose there are the two independent, uncertain and high conflict resource $B_{1}$ and $B_{2}$ in the same identification framework and the two general basic probability assignment function $m_{1}(\bullet)$ and $m_{2}(\bullet)$, and the DSmT combining rules $m_{M^{f}}(\bullet) \equiv m(\bullet) \stackrel{\Delta}{=}\left[m_{1} \oplus m_{2}\right](\bullet)$ is defined as:

$$
\forall A \neq \phi \in D^{U} m_{M^{f}(U)}(f) \stackrel{\Delta}{=}\left[m_{1} \oplus m_{2}\right](A)=\sum_{\substack{X_{1}, \cdots X_{k} \in D^{U} \\\left(X_{1} \cap \cdots \cap X_{k}\right)=A}} \prod_{i=1}^{k} m_{i}\left(X_{i}\right) \text { in which, } m_{M^{f}(U)}(\phi)=0
$$

\section{Fusion Fault Diagnosis Model}

The paper fusion the Rough Sets Theory and DSmT for fault diagnosis, firstly the uncertain and incomplete test data is preprocessed and condition attribution reduction and attribution value reduction to get the reduced decision table. Then, the basic probability assignment is calculated and the reduced condition attribution significance is calculated and normalized to get the evidence reasoning results. Finally, the evidence reasoning results are applied the DSmT combine rules to get the fault diagnosis results. The detail fault diagnosis process can see Fig. 1.

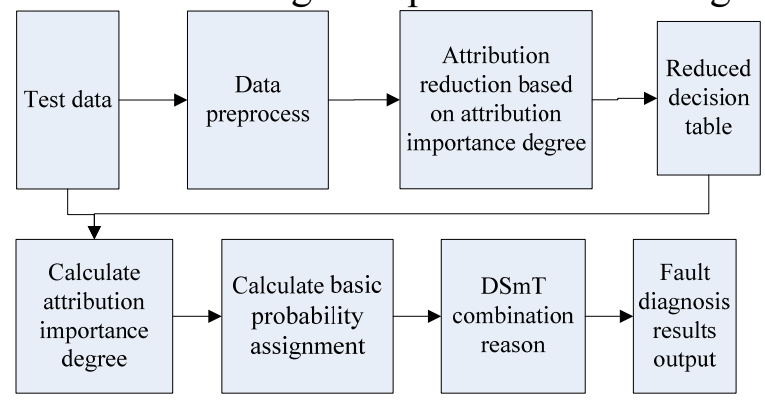

Fig.1 Fusion Fault Diagnosis process

\section{Application Example}

The paper discusses some airborne radio equipment fault diagnosis, the test data is extracted as fault examples and the above method is applied to get the diagnosis results. Table1 gives the test data of the familiar fault phenomenon "airborne radio", in which the fault symptom is expressed attribution reduction $C=\left\{+5 \mathrm{~V}\right.$ voltage $C_{1}$, UUT receiver voltage $C_{2}$, UUT receiver power $C_{3}$, UUT sender voltage $C_{4}$, UUT sender voltage $C_{5}, 1553 \mathrm{~B}$ bus $\left.C_{6}\right\}$, the decision attribution $D=\left\{d_{1}, d_{2}, d_{3}\right\}$, " $d_{1}$ "is fault synchronous module, " $d_{2}$ " is fault front panel module, " $d_{3}$ " is fault master control 
microcomputer module. The 8 times test fault data is extracted in Table1, in which the data from the 1st time to the 6th time is diagnosis sample, the 7 th time data is the normal sample, the 8th time data is the verified sample. Through the equipment normal work interval, the above data can discretizated and the original decision table is gotten.

Table 1 fault samples and original decision table

\begin{tabular}{cccccccccccccc}
\hline & $C_{1}$ & \multicolumn{1}{c}{$C_{2}$} & \multicolumn{2}{c}{$C_{3}$} & $C_{4}$ & \multicolumn{1}{c}{$C_{5}$} & $C_{6}$ & $D$ \\
\hline$(1)$ & 5.1 & 1 & 27.5 & 1 & 25 & 0 & 22.5 & 0 & 120 & 0 & 50 & 0 & $d_{1}$ \\
$(2)$ & 5.0 & 1 & 28.0 & 1 & 35 & 0 & 27.5 & 1 & 180 & 0 & 35 & 0 & $d_{1}$ \\
$(3)$ & 5.25 & 2 & 22.5 & 0 & 15 & 0 & 28.5 & 1 & 500 & 2 & 30 & 0 & $d_{2}$ \\
$(4)$ & 4.85 & 0 & 30.5 & 2 & 35 & 0 & 28.5 & 1 & 250 & 1 & 110 & 2 & $d_{2}$ \\
$(5)$ & 5.0 & 1 & 26.5 & 1 & 100 & 2 & 25.0 & 0 & 170 & 0 & 210 & 2 & $d_{3}$ \\
$(6)$ & 4.7 & 0 & 23.0 & 0 & 20 & 0 & 27.0 & 1 & 120 & 0 & 220 & 2 & $d_{3}$ \\
$(7)$ & 0 & 0 & 0 & 0 & 0 & 0 & 0 & 0 & 0 & 0 & 0 & 0 & normal \\
$(8)$ & 5.2 & 2 & 24.5 & 0 & 70 & 1 & 27.5 & 1 & 110 & 0 & 20 & 0 & $d_{2}$ \\
\hline
\end{tabular}

Calculate the discernibility matrix of the previous 6 time test samples, and the relative core $=\left\{C_{4}, C_{6}\right\}$. In the discernibility matrix, the all including core sets is extracted, it is found that the sets also contain the condition attribution " $C_{1}$ ", " $C_{2}$ ", " $C_{3}$ " and " $C_{5}$ ". Then, calculate the appearing their times through the attribution importance degree algorithm, we can get the relative reduction $R E D_{1}=\left\{C_{1}, C_{4}, C_{6}\right\}$ 和 $R E D_{2}=\left\{C_{2}, C_{4}, C_{6}\right\}$ and the reduced decision table is Table2.

Table2 the reduction table

\begin{tabular}{|c|c|c|c|c|c|c|c|}
\hline \multirow{2}{*}{ No. } & \multicolumn{3}{|c|}{$R E D_{1}$} & \multicolumn{3}{|c|}{$R E D_{2}$} & \multirow{2}{*}{$D$} \\
\hline & $C_{1}$ & $C_{4}$ & $C_{6}$ & $C_{2}$ & $C_{4}$ & $C_{6}$ & \\
\hline (1) & 1 & 0 & 0 & 1 & 0 & 0 & $d_{1}$ \\
\hline (2) & 1 & 1 & 0 & 1 & 1 & 0 & $d_{1}$ \\
\hline (3) & 2 & 1 & 0 & 0 & 1 & 0 & $d_{2}$ \\
\hline (4) & 0 & 1 & 2 & 2 & 1 & 2 & $d_{2}$ \\
\hline (5) & 1 & 0 & 2 & 1 & 0 & 2 & $d_{3}$ \\
\hline (6) & 0 & 1 & 1 & 0 & 1 & 2 & $d_{3}$ \\
\hline (7) & 0 & 0 & 0 & 0 & 0 & 0 & normal \\
\hline
\end{tabular}

Then, take $C_{1}, C_{4}, C_{6}$ or $C_{2}, C_{4}, C_{6}$ as $r_{1}, r_{2}, r_{3}$ or $R_{1}, R_{2}, R_{3}$ to combine the evidence $r$ or $R$, and take $d_{1}, d_{2}, d_{3}$ and normal as frame of discernment $\Theta 1, \Theta 2, \Theta 3, \Theta 4$. The basic probability assignment of $R E D_{1}=\left\{C_{1}, C_{4}, C_{6}\right\}$ is gotten as Table 3 .

Table 3 the basic probability assignment

\begin{tabular}{ccccc} 
& $\Theta 1$ & $\Theta 2$ & $\Theta 3$ & $\Theta 4$ \\
\hline$r_{1}$ & 0 & 1 & 0 & 0 \\
$r_{2}$ & 0.25 & 0.5 & 0.25 & 0 \\
$r_{3}$ & 0.5 & 0.25 & 0 & 0.25
\end{tabular}

Calculate the attribution importance degree of $R E D_{1}=\left\{C_{1}, C_{4}, C_{6}\right\}$ relative to $D$, we can get $S G F\left(C_{1}, D\right)=2 / 7, S G F\left(C_{4}, D\right)=2 / 7, S G F\left(C_{6}, D\right)=4 / 7$, then normalize the attribution importance degree to get the basic probability assignment $\lambda_{1}=0.25, \lambda_{2}=0.25, \lambda_{3}=0.5$, the evidence combined results can be gotten as Table4. Use the same way the $R E D_{2}=\left\{C_{2}, C_{4}, C_{6}\right\}$ combined results is gotten as Table4.

Table4 $R E D_{1}$ and $R E D_{2}$ combined results

\begin{tabular}{ccccc}
\hline & $\Theta 1$ & $\Theta 2$ & $\Theta 3$ & $\Theta 4$ \\
\hline$r$ & 0.3125 & 0.25 & 0.0625 & 0.125 \\
\hline$R$ & 0.214 & 0.357 & $0 . .214$ & 0.214
\end{tabular}

DSmT combined rules are applied to calculate the Table4 results, and the results are in Table6.

Table6 DSmT combined results

\begin{tabular}{cccccccc}
\hline & $d_{1}$ & $d_{2}$ & $d_{3}$ & $d_{1} \cap d_{2}$ & $d_{1} \cap d_{3}$ & $d_{2} \cap d_{3}$ & normal \\
\hline$r+R$ & 0.16 & 0.19 & 0.053 & 0.17 & 0.076 & 0.067 & 0.027 \\
\hline
\end{tabular}


Through contrasting the fault diagnosis results, it is likely to be " $d_{2}$ ", and it is accordance with the real test result. So the above method is valid.

\section{Summary}

The paper gives one fusion method based on Rough Sets Theory and DSmT, which has some processing advantage of uncertain information. The application example verified its validity. It providers the new approach of the information fusion methods for equipment fault diagnosis.

\section{References}

[1] Xu Tingxue. Equipment Comprehensive Support Engineering Theory and methods[M].Beijing: Weapons industry Press,2009(in Chinese)

[2] Z. Pawlak. Rough sets [J]. International Journal of Information and Computer Science, 1982,11:341-356

[3] Miao Duoqian, Li Daoguo. Rough Sets Theory Algorithms and Applications[M]. Beijing: Qinghua University Press, 2008(in Chinese)

[4] He You, Wang Guohong, Guan Xin. Multisensor information fusion with Applications[M].Beijing: Publishing house of electronics industry, 2010(in Chinese)

[5] Application of DSmT to Target Identity Identification[J].Journal of Air Force Rader Acadmy,2010,8(24):251-253(in Chinese) 